\title{
A new health services platform for patient centred and personalised care
}

BMJ 2014; 349 doi: http://dx.doi.org/10.1136/bmj.g6842 (Published 18 November 2014)Cite this as: BMJ 2014;349:g6842

- $\quad$ Article

- $\quad$ Related content

- Metrics

- $\underline{\text { Responses }}$

1. Shahid Ali, general practitioner principal and director Dynamic Health Systems,

2. Axel Schulte, chief executive officer,

3. Richard Pope, clinical director,

4. Douglas Muir, chief technical officer 2 Author affiliations

1. shahid.ali@bradford.nhs.uk

We fully support Bisognano and Schummers's well presented and timely article. 1 The 2002 Wanless report made us realise that without a change in approach the NHS would become financially challenged and that we needed a way to engage patients. We decided to use an evidence based, patient centred approach to enable patients with long term conditions to take control of their health through shared decision making.

Patients with long term conditions were seen in care planning clinics where they could speak about their problems, including social, health, and economic ones. Patients set their goals and action plans - guided and coached by the clinician — and took home a print out of their care plans. Analysis showed a $40 \%$ reduction of service utilisation costs in these patients. $\underline{\underline{4}} 4$ This test of the care planning approach shows that "flipping healthcare" works.

However, large scale deployment of this approach presented organisational challenges. Turning digital, we incorporated the innovation into an internet delivered service called VitruCare, a scalable and integrated digital health services platform that can present different service packages to suit the patient. $\underline{5} \mathbf{6}$ Patients can set goals, action plans, and see real time changes in their health status while connected to and coached by their clinician. Early outcome data show improvements in weight, blood pressure, and glycosylated haemoglobin through lifestyle changes. This type of approach provides a route for individuals, families, and communities to engage and enables the cultural change in approach that the NHS desperately needs to be reinvigorated and to thrive. We believe that patient centred and personalised care will become the norm across the health service - engaging and activating patients, as well as enabling proactive management and immediacy to deliver improved outcomes in both health and healthcare.

\section{Notes}

Cite this as: $B M J$ 2014;349:g6842

\section{Footnotes}

- Competing interests: We are all directors of Dynamic Health Systems, which created and designed VitruCare on the basis of clinical experience and patient participation.

- Full response at: www.bmj.com/content/349/bmj.g5852/rr/779957. 


\section{References}

1.

Bisognano M, Schummers D. Flipping healthcare: an essay by Maureen Bisognano and Dan

Schummers.BMJ2014;349:g5852. (3 October.)

FREE Full Text

2. $\leftrightarrows$

Right Care Shared Decision Making Programme. Measuring shared decision making: a review of research evidence. 2012. Cambridge. http://sdm.rightcare.nhs.uk/.

3.

Ali SM, Colletta J, Pope R. Self care planning. Health Serv J2010 Apr

22. www.hsj.co.uk/resource-centre/your-ideas-and-suggestions/self-careplanning/5013256.article\#.VGX9HDRdNpd.

4. $\leftrightarrows$

Ali SM, Colletta J, Pope R. QIPP and care plans revisited. Health Serv J2011 May

19. www.hsj.co.uk/resource-centre/performance-healthcheck/qipp-and-care-plans-for-long-termconditions-revisited/5028822.article.

5. $\lrcorner$

Patients and Information Directorate, NHS England. VitruCare online service. Supporting patients with long-term conditions. Transforming participation in health and care. Gateway Reference No 00381. 2013. www.england.nhs.uk/wp-content/uploads/2013/09/trans-part-hc-guid1.pdf.

6.

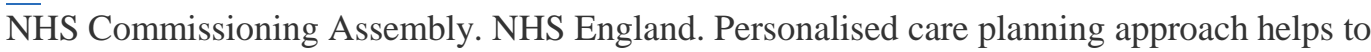
improve patient outcomes in Bradford. Case study.

2014. https://www.learnenv.england.nhs.uk/pinboard/download/id/231. 